\title{
An improved fast transform algorithm based on adaptive hybrid channel allocation in TSCH networks
}

\author{
Junxin Gu, Yong Xu, Yujun Zhu*, Fan Mei, Longlong Kang and Yiyang Liu
}

\author{
* Correspondence: zhuyujun@mail. \\ ahnu.edu.cn \\ School of Computer and \\ Information, Anhui Normal \\ University, 241002 Wuhu, China
}

\begin{abstract}
Time Slotted Channel Hopping (TSCH) is one of the five access behavior technologies defined in the IEEE 802.15.4e standard. Research shows that the wireless sensor network with single dynamic and static channel allocation in $\mathrm{TSCH}$ mode has higher performance. The clogging rate results in unnecessary energy waste. Therefore, this paper proposes an adaptive hybrid channel allocation strategy (AHCA) to measure the link quality change of wireless sensor networks in TSCH mode. The strategy estimates the part of the channel quality and determines whether the node wants to transmit on this channel and the optimal channel for node information transmission. According to the change of the amount of information transmitted, the hybrid channel allocation scheme of the dynamic and static channel ratio is adaptively adjusted to reduce the energy consumption of the information transmission process. This article performs experimental simulation on Contiki-NG. The experimental results show that the proposed scheme has shorter transmission time and lower congestion rate than the single dynamic static channel allocation wireless sensor network. The more reasonable and effective measurement, analysis and use of channel resources can fundamentally increase the amount of information transfer in wireless sensor networks per unit time, and thus extend the lifetime of wireless sensor networks.
\end{abstract}

Keywords: IEEE 802.15.4e, Wireless sensor network, Hybrid channel allocation (HCA), Time Slotted Channel Hopping (TSCH), Resource Scheduling Algorithm

\section{Introduction}

With the emergence of emerging industrial Internet of Things application scenarios on a large scale, IEEE 802.15.4 is no longer able to cope with these new scene requirements. Therefore, IEEE 802.15.4e-2012 [1] emerges at the right moment, aiming to meet the requirements of emerging industrial Internet of Things scenarios. IEEE 802.15.4e-2012 is an improvement of THE IEEE 802.15.4 protocol standard, introducing a time-slot channel frequency-hopping mode. Compared with traditional network, IEEE 802.15.4e standard, which introduces time synchronization protocol, emphasizes time synchronization and reduces power

(c) The Author(s). 2020 Open Access This article is licensed under a Creative Commons Attribution 4.0 International License, which permits use, sharing, adaptation, distribution and reproduction in any medium or format, as long as you give appropriate credit to the original author(s) and the source, provide a link to the Creative Commons licence, and indicate if changes were made. The images or other third party material in this article are included in the article's Creative Commons licence, unless indicated otherwise in a credit line to the material. If material is not included in the article's Creative Commons licence and your intended use is not permitted by statutory regulation or exceeds the permitted use, you will need to obtain permission directly from the copyright holder. To view a copy of this licence, visit http://creativecommons.org/licenses/by/4.0/. 
consumption. Wireless sensor network (WSN) due to low cost and flexibility so is better than that of wired networks in nature, but the wireless sensor network has low reliability, because the information transmission process prone to conflicts and external disturbance, and the pattern of TSCH IEEE 802.15.4e using channel hopping technology against interference and selective fading channel, the channel by channel frequency hopping and proper scheduling, which improve the reliability of information transmission.

The power of wireless sensors usually comes from the tiny batteries they carry around. In reality, it is very inconvenient to replace or charge the battery. Once the energy of the sensor node is exhausted and cannot be replenished in time, the node will fail. The accumulation of failure nodes will reduce the sensitivity of the sensor network and lead to the failure of data transmission. From the perspective of current research, energy consumption has always been the core problem faced by wireless sensor networks. Therefore, from the perspective of reducing energy consumption, increasing the life cycle of wireless sensor networks conforms to the development trajectory of the current era of wireless sensor networks.

The study proposes a minimum timetable (TSCH-Minimal) scheme [1] to refine the network time slot length as much as possible. In order to increase the efficiency of data packet transmission and system fairness, a dedicated TSCH unit and a shared TSCH time slot are proposed to coexist with the same schedule [2]. Some authors proposed the Orchestra scheme [2]. Nodes are autonomously scheduled locally, relying on the existing network stack to maintain scheduling, and there is no signaling overhead, but the algorithm requires that the ordinary nodes have enough energy and are very dependent on network density. It has also been proposed to handle unpredictable traffic through shared access and burst arrival [3]. Atis proposed a PRR-based channel quality assessment algorithm for downstream channel selection [4]. The channel evaluation can be used to distinguish the interference degree of the channel, reduce the retransmission of data packets, reduce the congestion rate, and reduce the retransmission time. However, since all channels need to scan the channel multiple times, this method will cause more energy consumption in the evaluation channel. Ojo [5] proposed an energy-centralized scheduling method to cope with the problem of network transmission failure and information transmission failure to increase the congestion rate. Using the extra heuristic efficiency scheduler and the Vogel approximation-based scheduling to solve the defects in the scheduling algorithm, and finally to achieve better information throughput in the wireless sensor network. Gomes [6] presents a distributed method for channel quality estimation of dedicated nodes. Using a dynamic channel allocation (DCA) strategy, the dedicated node detects the channel quality so that the network node can decide whether to change the channel and the best channel to use. The method is based on a hardware estimator and may overestimate the link quality when encountering a high-packet error rate. The time slot frame length is optimized by mountain climbing [7]. Through the simulation experiment results of cubic regression equation, the transmission blocking rate and transmission time can be improved by optimizing the time slot frame length; Adnan proposes a distributed method for channel quality estimation of dedicated 
nodes in wireless sensor networks [8], which uses dedicated nodes to monitor channel quality, which identifies low-quality channels by received signal strength indicator (RSSI) and link quality indicator (LQI), and predicts the best channel. Literature shows A centralized MABO-TSCH channel scheduling algorithm [9], the overall use of dynamic programming channels, nodes share channels, while the blacklist mechanism, the poor quality of the channel into the blacklist, in the better quality of the channel for information transmission; excluding some unnecessary channels from the frequency hopping sequence can improve the reliability of communication while maintaining the benefits of TSCH. Yang proposes an adaptive frequency hopping time slot channel (A-TSCH) [10], designing a channel access method. Elsts presents an adaptive channel selection strategy [4] for TSCH networks. Sun designed a block chain system full function node optimization configuration algorithm based on the maximization of transaction throughput [11], so that the network can reach the optimal performance analysis and deployment of the optimal communication node. In the case of serious interference, the standard TSCH does not have enough scheduling slots to reliably transmit all data packets, while the adaptive approach makes the system more reliable, in which PRR-based channel quality assessment and downstream driver channel selection show the best results. Although these methods in the literature have balanced the node load and network energy in a sense, they can only partially reduce the occurrence of partial congestion in the network and partially reduce the transmission time of information in the network.

So far, no one considers the hybrid channel allocation strategy into the TSCH network. On the basis of predecessors, in this paper, a scheme combining adaptive scheduling and hybrid channel allocation is proposed to reduce the end-to-end delay time of information in the channel, reduce the channel congestion rate, and increase the reliability and duty ratio of information transmission in wireless sensor networks under the circumstances of high-interference environment and frequent communication connection changes.

The subsequent arrangement of the paper is as follows: Section II introduces the related research of TSCH. Section III introduces the adaptive HCA scheme. Section IV is the experimental simulation and results, and finally draws the experimental conclusion.

\section{Related work}

In order to reduce the information transmission time and increase the efficiency of information transmission in wireless sensor networks, an adaptive HCA scheme (AHCA) is introduced. The AHCA scheme is an algorithm that combines the mixed channel allocation strategy with the dedicated node technology and divides the channel in the TSCH network into two parts: public and private, estimates the quality of public channel, pauses the transmission of information for the channel with poor quality, and continues to transmit information for the channel with better quality. In order to reduce the collision of information in wireless channel, reduce the energy consumption, and increase the lifetime of wireless sensor network, the special channel strategy is adopted for some nodes which send more information. 


\subsection{Channel selection}

In a TSCH network, a node typically uses three inputs to determine the channel to be used for a particular transmission [11]: the absolute time slot (ASN) value of the network, the scheduled channel offset $\left(\mathrm{CH}_{\text {offset }}\right)$, and the pseudo-random frequency hopping sequence (HS), stored as a lookup table:

$$
\text { channel }=H S\left[\left(A S N+C_{o f f s e t}\right) \%\|H S\|\right]
$$

Where ASN represents the total number of time slots from the beginning of the TSCH network, ASN $=\left(k^{*} s+t_{s}\right), k$ is the time slot period, $s$ is the time slot frame size, $t_{s}$ representing the time slot. (Where $0 \leq t_{s} \leq s-1,0 \leq \mathrm{CH}_{\text {offset }} \leq||$ HS || ) where the IEEE 802.15.4e network running at the $2.4 \mathrm{GHz}$ has a total of 16 (11-26) channels.

Assuming that when 6 channels are fixed, there are 10 channels left for information transmission, the channel selection is as shown in the Fig. 1.

$$
\mathrm{HS}=\{16,17,23,18,26,15,25,22,19,11\} \begin{aligned}
C H_{\text {offset }} & =H S[(4+1) \bmod 10] \\
& =H S[5] \\
& =15(\text { channel } 15)
\end{aligned}
$$

In this example, an arbitrary vector of channel $\mathrm{V}$ is used, and slot 3 within the slot frame is characterized by $\mathrm{CH}_{\text {offset }}=1$. The increment counter ASN in (1) causes slot 3 to use a different channel for each slot frame period.

TSCH networks are deterministic in their scheduling regardless of the lossy nature of the wireless medium. The TSCH schedule can be represented by a $2 \mathrm{D}$ matrix, with the width being the number of slots in the slot frame and the height being the number of frequencies available, as shown in Fig. 1.

\subsection{Channel quality assessment}

There are many ways to evaluate the channel quality in wireless sensor networks. Reference uses the value of RSSI [11] to evaluate the channel quality. As shown in formula (2), the measured channel quality can be obtained by using exponential smoothing (SES) technique to calculate the quality estimate (QE) of each channel.

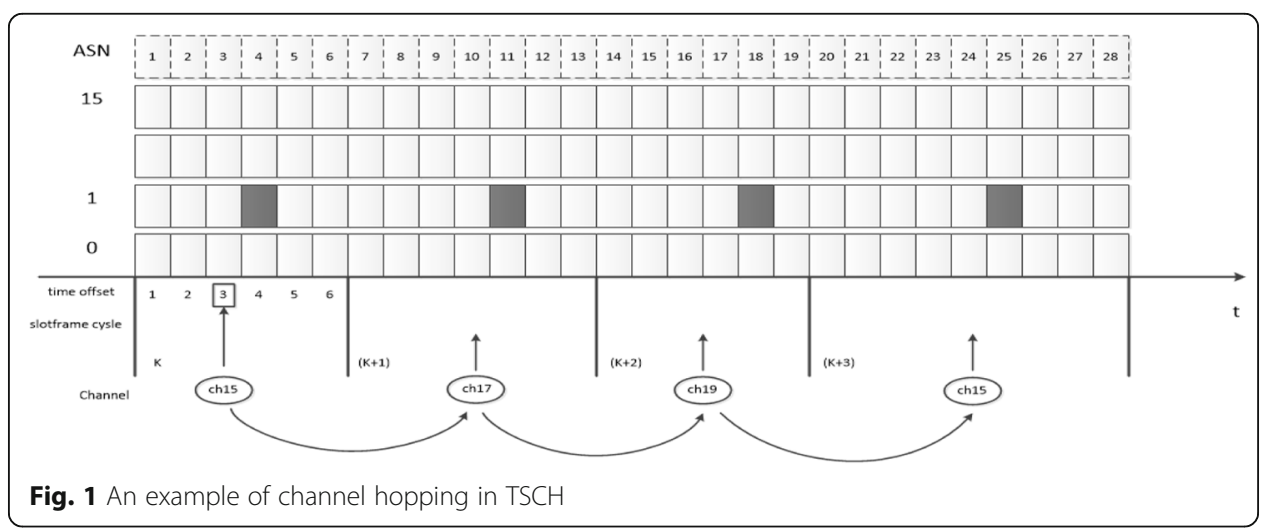




$$
\begin{aligned}
& Q E[c]_{k}=\left\{\begin{array}{c}
N F[c]_{0} k=0 \\
\alpha Q[c]_{k-1}+(1-\alpha) N F[c]_{k}
\end{array} \quad k \in[1, \infty)\right. \\
& c \in[11,26], \quad \alpha \in[0,1]
\end{aligned}
$$

$\mathrm{QE}[\mathrm{c}]_{\mathrm{k}}$ represents an estimate of the channel quality obtained after the transmission of the $k$ th message, the initial estimate $\mathrm{QE}[\mathrm{c}]_{0}$ is specified as $\mathrm{NF}[\mathrm{c}]_{0}$, which is the first estimate of the channel quality. According to Eq. (2), the following estimates up to the $k$ th mass can be obtained in turn. The next estimate of $K$ is computed using the nearest channel quality and is affected by the coefficients.

The next estimate of $k$ is calculated using $\alpha Q E[c]_{k-1}$ and the most recent channel quality $\mathrm{NF}[c]_{k}$ and is affected by the $\alpha$.

\section{Adaptive HCA scheme (AHCA)}

When adaptive AHCA algorithm is used, normal communication between nodes can be satisfied in high-congestion environment. Transmit information between nodes, the sending node can choose high quality in the local channel, which decided to channel and when to send information, considering the frequent node of information transmission, if the information is frequent in high-quality transmission channel, can cause congestion, at this time we need to give information flow into shorter package, make the information transmission has a shorter average time.

Can be based on the theory of the proposed algorithm steps are as follows: ready to send information of nodes in the network to listen to a better quality of channel according to the size of the flow of information between the nodes in the shared channel, or fixed channel transmission, as the number of the fixed channel and the change of the amount of information sharing channel variable; this reduces the amount of time required for information transmission, saves network energy consumption, and further increases the network life cycle.

\subsection{Performance analysis of HCA}

HCA is a combination of fixed channel allocation (FCA) and dynamic channel allocation (DCA), so the performance of HCA has the common characteristics of FCA and DCA [12-14].

In FCA, we assume that each node in the network has an equal amount of information transmission, and the probability of channel congestion satisfies the following formula (3)

$$
B_{F C A}=\left(a^{N c} / N_{c} !\right) / \sum_{i=0}^{N c} a^{i} / i !
$$

$\alpha$ is the average amount of information transferred per node; $N_{c}$ is the number of channels available for each node under FCA conditions.

In DCA, we assume that all channel assignments are independent and have the same priority, the first assignment does not need to distinguish channel quality, only the number of occupied channels, and the congestion rate of DCA should be as follows (4) 


$$
B_{D C A}=\sum_{x=0}^{N} G(x) P(x)
$$

Where $\mathrm{G}(\mathrm{x})$ is the probability of having an idle channel and $\mathrm{P}(\mathrm{x})$ is the probability that $\mathrm{x}$ pieces of information are being transmitted in the node. From the above formula, we can see that if we want to obtain the blocking rate, we first need to find $G(x)$ and $P$ (x).

We assume that in a common channel, the amount of information transmitted between nodes is balanced and the probability of the channel being occupied is known, in which case $\mathrm{G}(\mathrm{x})$ satisfies a binomial distribution (5)

$$
G(x)=\frac{N !}{x !(N !-x)} n^{N-x}(1-n)^{x}
$$

In the DCA network, the average occupancy rate of the common channel is set as $N$, the total number of nodes is $M$, the total number of channels is $N$, and the total amount of information carried by the network is $A$, then the average amount of information carried by each channel in each node is A/MN. Without considering the influence of other nodes, given any node, the probability of channel idle is $1-\mathrm{a} /(\mathrm{MN})$. A typical $K=6$ star topology network [15], because of the state of channel multiplexing, when a channel is used by a node, it is set that adjacent $K-1$ nodes will disable the channel. This typical value will be adopted in the subsequent research of this paper. In order to have the same priority of channels, it is assumed that the use of each channel is independent under certain conditions, and the average occupancy rate of the common channel can be obtained (6):

$$
n=1-\left[1-A /(M N)^{K}\right]
$$

Next, we can get formula (7):

$$
F(x)=\sum_{y=x}^{N} G(y)=\sum_{y=x}^{N} \frac{N !}{x !(N !-x)} n^{N-y}(1-n)^{y}
$$

If the energy transmission quantity of nodes in the system is balanced, the probability $\mathrm{P}(\mathrm{x})$ of the channel occupied is calculated, assuming that the number of nodes in the network is $N$, the arrival rate of node information is $\alpha$, and the departure rate of node information is $\beta$, then combining

$$
\begin{aligned}
& a P[x]=(x+1) b P(x+1) \\
& \sum_{i} P(i)=1
\end{aligned}
$$

So, we may obtain 


$$
P(x)=\frac{(a / b)^{x}}{x !} P(0)
$$

In combination with formula (4), (7) and (8), it can be obtained (9):

$$
B_{D C A}=\frac{\sum_{x=0}^{N} G(x)(a / b)^{x} / x !}{\sum_{x=0}^{N} F(x)(a / b)^{x} / x !}
$$

Because HCA can be viewed as a combination of DCA and FCA, congestion occurs only when the fixed and dynamic channels of a node are used up in HCA system. We compare the congestion rate of HCA with that of DCA. The congestion rate of HCA can be calculated by the product of the fixed channel congestion rate and the overflow dynamic channel congestion rate. We use $B_{\mathrm{HCA}}\left(N_{H}, A\right)$ here to represent the blocking rate. $N_{H}$ represents the number of channels available for the HCA.

$$
\begin{aligned}
& B_{H C A}\left(N_{H}, A\right) \\
& =B_{F C A}\left(\frac{N_{F}}{k}, \frac{A}{M}\right) B_{D C A}\left[N_{D}, A B_{F C A}\left(\frac{N_{F}}{k}, \frac{A}{M}\right)\right]
\end{aligned}
$$

Therefore, the ratio of fixed channel to dynamic channel in HCA is $Y=N_{F} / N_{D}$; At this point, and then set $a=A / M$, we can rewrite the (10) to formula (11):

$$
\begin{aligned}
& B_{H C A}(N H, A) \\
& =B_{F C A}\left[\frac{Y N}{(1+Y) K}, a\right] B_{D C A}\left[\frac{N}{(1+Y)}, A B\left(\frac{Y N}{(1+Y) K}, a\right)\right]
\end{aligned}
$$

\subsection{Hybrid channel assignment ratio}

There is a balance between the number of common channels that can be freely allocated and the amount of information transmitted between nodes. When the amount of information transmitted is small, HCA strategy has a generally low blocking rate; when the amount of information transmitted is large, the blocking rate of HCA will increase. Therefore, when the number of common channels is determined, there will be a unique and determined channel allocation ratio for different information transmission networks, so that the network congestion rate is minimized. For example, when the amount of information is $A$, the optimal channel allocation ratio $Y$ can be achieved, so the number of channels needs to be allocated at any time according to the change of the amount of information to achieve the optimal channel allocation ratio, and the optimal channel allocation ratio can reduce the collision between channels, thereby reducing the energy consumption of nodes and increasing the life of wireless sensor networks. 


\subsection{AHCA algorithm mechanism}

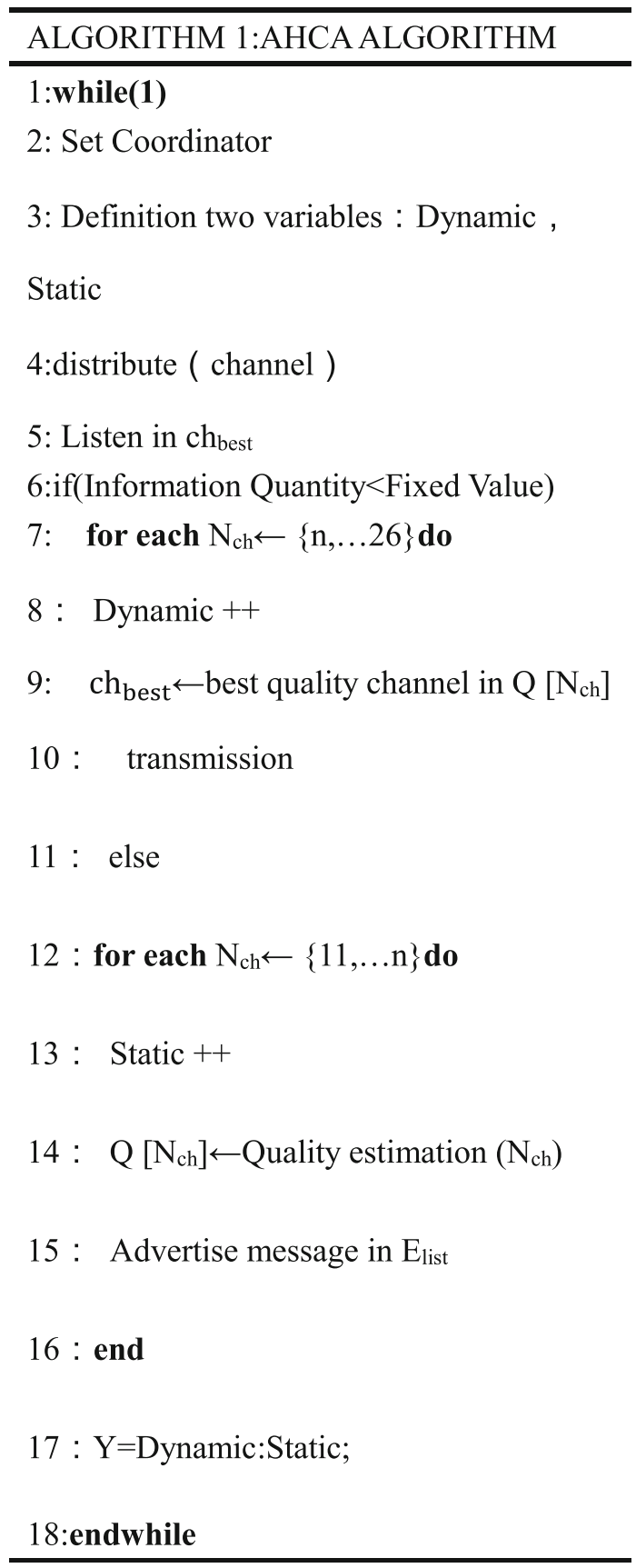

The fourth line of the algorithm divides the dynamic and static channels. Lines 6-10 show that when the amount of information is less than a certain value, the information between nodes is transferred in the dynamic channel, and lines 12-16 show that when the amount of information between nodes is greater than a certain value, it is transferred in the static channel. Line 17 shows the division of dynamic and static channel proportion. Generally speaking, nodes will select any channel in the dynamic channel for information transmission, but the quality of each channel in the dynamic channel will be different due to the channel blocking, so the information transmission time is 
different. The algorithm shows the mechanism of selecting the dynamic and static channels. Using the special node to evaluate the channel quality can achieve the optimal network information transmission time.

\section{Simulation results and discussion}

In this paper, simulation experiments are carried out on the Cooja simulator: 10, 50, 98 ordinary nodes are deployed in the range of 100 m*100 m, respectively, and one coordinator node represents the deployment of nodes in sparse, ordinary, and dense networks respectively. Under the model of AHCA, DCA and Orchestra, several important indicators were considered: information transmission time, end-to-end delay time, reliability (PRR) [16], duty cycle, and channel congestion rate. End-to-end latency refers to the time it takes for a packet to travel over the network from one source to a destination, PRR refers to the ratio of successfully received packets to the total number of packets sent, and duty cycle refers to the ratio of the time the node is in the state of listening, sending and receiving.

Figure 2 is a topology diagram of node distribution information transmission, which contains a coordinator node and 10 ordinary nodes.

In the above experimental scenario, two parameters of the IOT application are changed: network density and transmission rate. Compared with the above three models, three groups of experiments were conducted to measure the impact of high-packet frequency, network density, and data volume on the performance of IOT applications. The first experiment is to change the network node density and measure the transmission time of the same number of packets. The second set of experiments tested the effects of changing the transmission rate on transmission reliability, end-to-end delay, and energy consumption in a dense network. The third experiment USES AHCA algorithm to monitor the influence of channel configuration ratio and information transfer amount on the congestion rate in dense network.

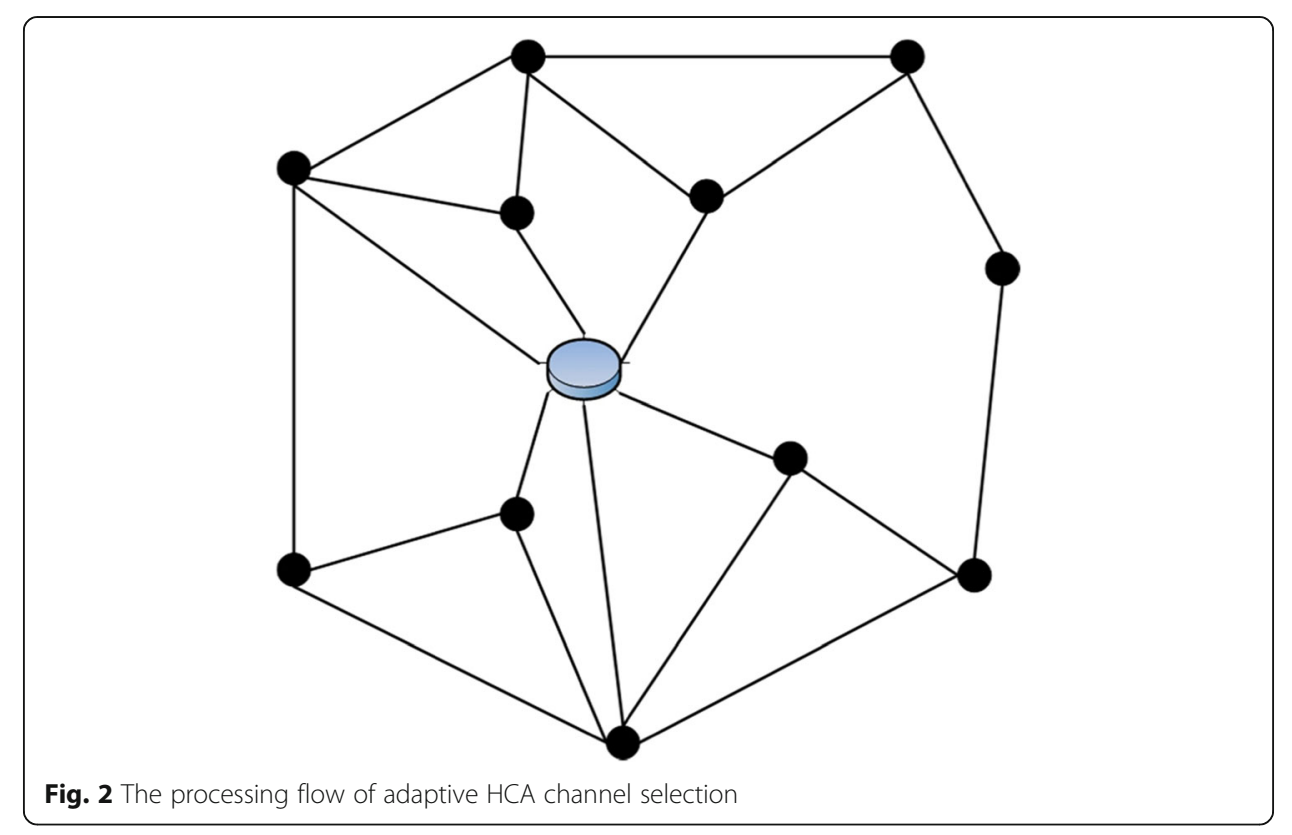




\section{Experiment 1-the influence of network density on transmission time}

In order to evaluate three different pass under the network density of the same time, under the condition of sparse, ordinary, dense set different number of data packets, Fig. 3 is based on the node distribution of Fig. 2, when the coordinator node use DCA algorithm, AHCA algorithm, and Orchestra method, transmission time changes over passing packet number of rendering. Figs. 3, 4, and 5, respectively, show the comparison time of information transmission of the three algorithms in the case of 20,50, and 98 nodes. It can be seen from Fig. 3 that the more packets, the shorter transmission time will be if AHCA algorithm is used than DCA algorithm. Meanwhile, the comparison shows that, within a certain range, when the data amount is the same, the higher the network density, the shorter the transmission time.

According to the node distribution in Fig. 2, when the coordinator node uses DCA algorithm, AHCA algorithm and Orchestra method, the time varies with the number of packets transferred. Figure 4 shows the comparison time of the three algorithms with 50 nodes. It can be seen from Fig. 3 that the more packets, the AHCA algorithm will have a shorter transmission time than THE DCA algorithm. When 500 packets are transferred, the AHCA algorithm needs about 105 s, the DCA algorithm 109 s, and the Orchestra algorithm $116 \mathrm{~s}$. Meanwhile, by comparing Figs. 4 and 5, it can be seen that the more nodes in the same range, the faster the data packet is transmitted.

\subsection{The second experiment-the influence of transmission rate on network performance}

To investigate the impact of application-level transmission rates on the reliability, latency, and energy of the scheduling algorithm evaluated, we ran the reference application scenario in a dense network of 98 nodes. At the beginning, necessary time is reserved to ensure the formation of the network, and then the DAG root [17] starts to send requests to the given node. We change the sending interval on the DAG root through different sending time intervals $(0.65 \mathrm{~s}, 1 \mathrm{~s}, 2 \mathrm{~s}, 5 \mathrm{~s}, 10 \mathrm{~s})$, and reruns the

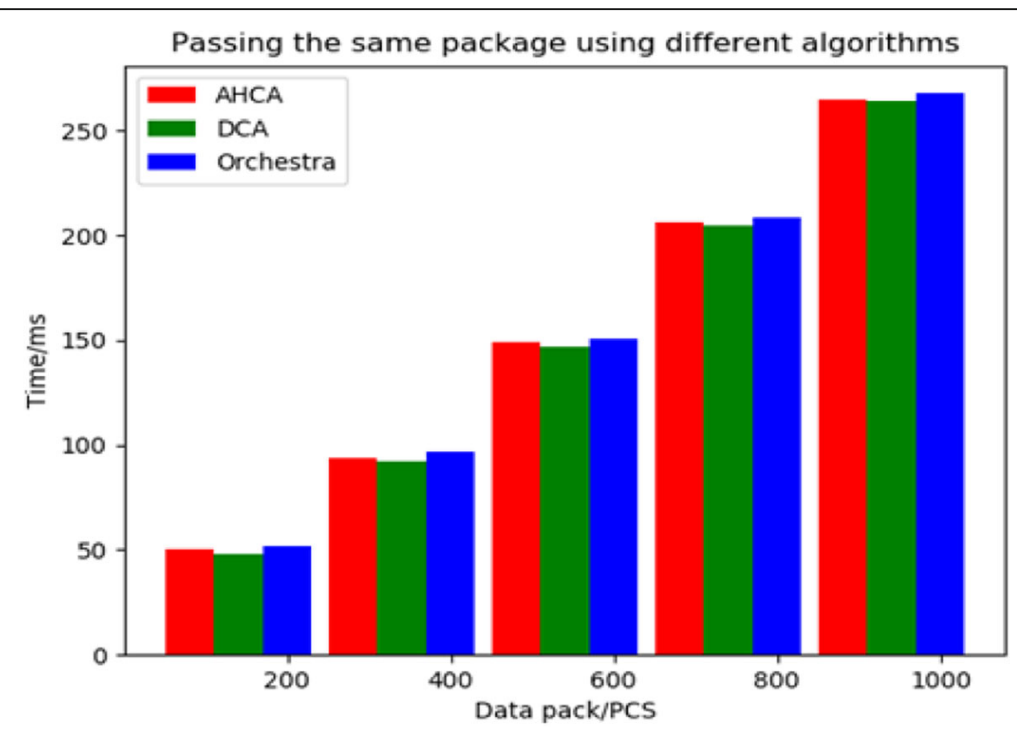

Fig. 3 An example of node deployment 


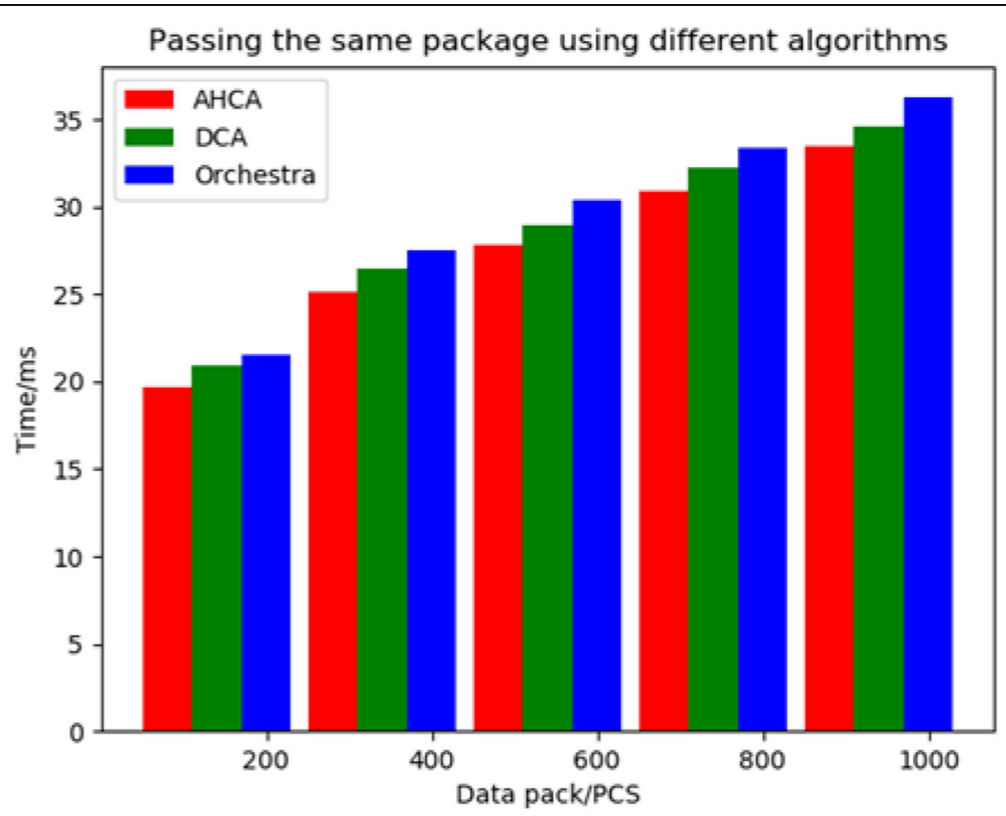

Fig. 4 Time to transmit the same messages with different algorithms(10 nodes)

experiment. Each experiment is repeated for 5 times, and the average result is shown in the figure below.

Figure 6 shows the average delivery ratio of Orchestra, AHCA, and DCA algorithms. It is observed that when the sending interval is greater than $2 \mathrm{~s}$, these three methods provide high reliability. However, when the sending interval is small, DCA decreases Orchestra by $27 \%$ and $28 \%$, respectively, while AHCA reduces by $25 \%$, due to the channel congestion rate caused by high-sending frequency. In fact, at this transmission rate, although the sender has started sending new requests, previous requests are still not

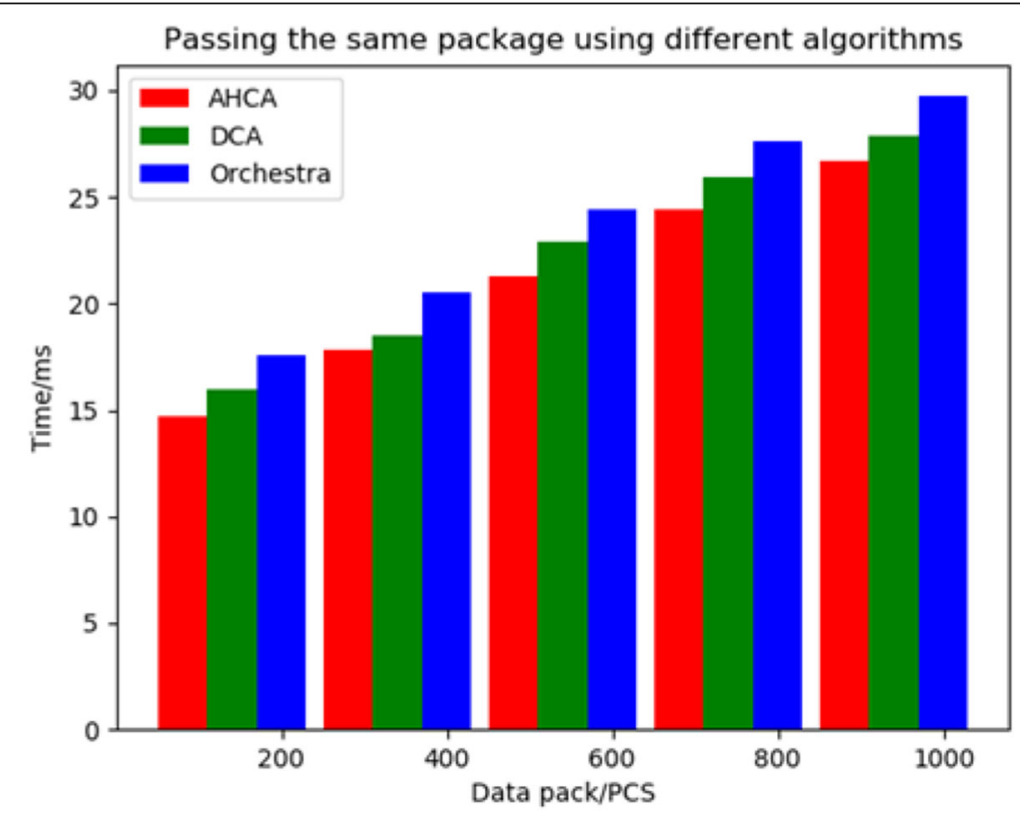

Fig. 5 Time to transmit the same messages with different algorithms(20 nodes) 


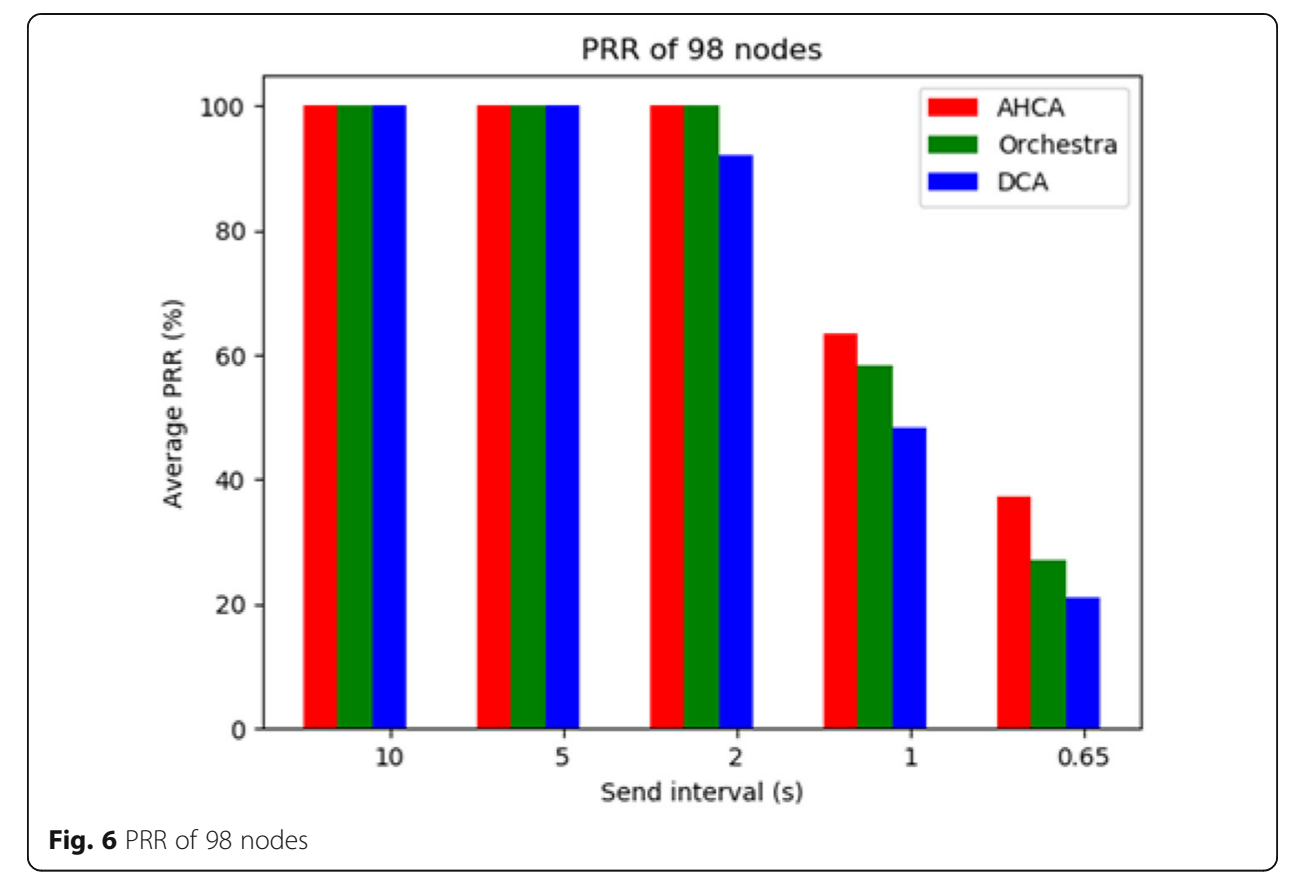

being responded. There is a solution to this by increasing the buffer size of the transfer at the expense of increasing the transfer latency, but this solution is affected by node performance and memory capacity.

Comparing the three scheme of PRR, one can find Orchestra and AHCA is more reliable than DCA; this can be explained by the Orchestra and the AHCA increase in the time slot frame the concept of priority, giving higher priority address, higher priority time slot frames, then is also planning a routing priority, allowing the division of priority, network to resynchronize, network transmission and routing maintenance, and DCA not set priority, may lead to a large amount of data of disposable inflows, and such information may be lost due to routing failure.

Although DCA is not as reliable as AHCA and Orchestra, it achieves better latency, as shown in Fig. 7, which can be explained by the small number of time slices for time slot frames in DCA. AHCA has larger time slot frames than DCA, and Orchestra has larger time slot frames, so DCA and AHCA have greater advantages in information transmission than Orchestra.

Finally, Fig. 8 shows the radio duty cycle of the three algorithms. The radio duty ratio of the three algorithms increases with the increase of transmission rate. Although both AHCA and Orchestra have larger time slot frames, they consume less energy than DCA, because most of the time slot intervals are arranged as sleep slots, thus consuming less energy.

\subsection{The third group of experiments-the influence of channel configuration ratio and information transmission amount on the clogging rate}

TSCH network has 16 channels at $2.4 \mathrm{GHz}$. Ratio and amount of information are needed in order to study the channel configuration for plugging rate. In the case of the number of nodes in a dense network (98), we realized the AHCA algorithm, the 


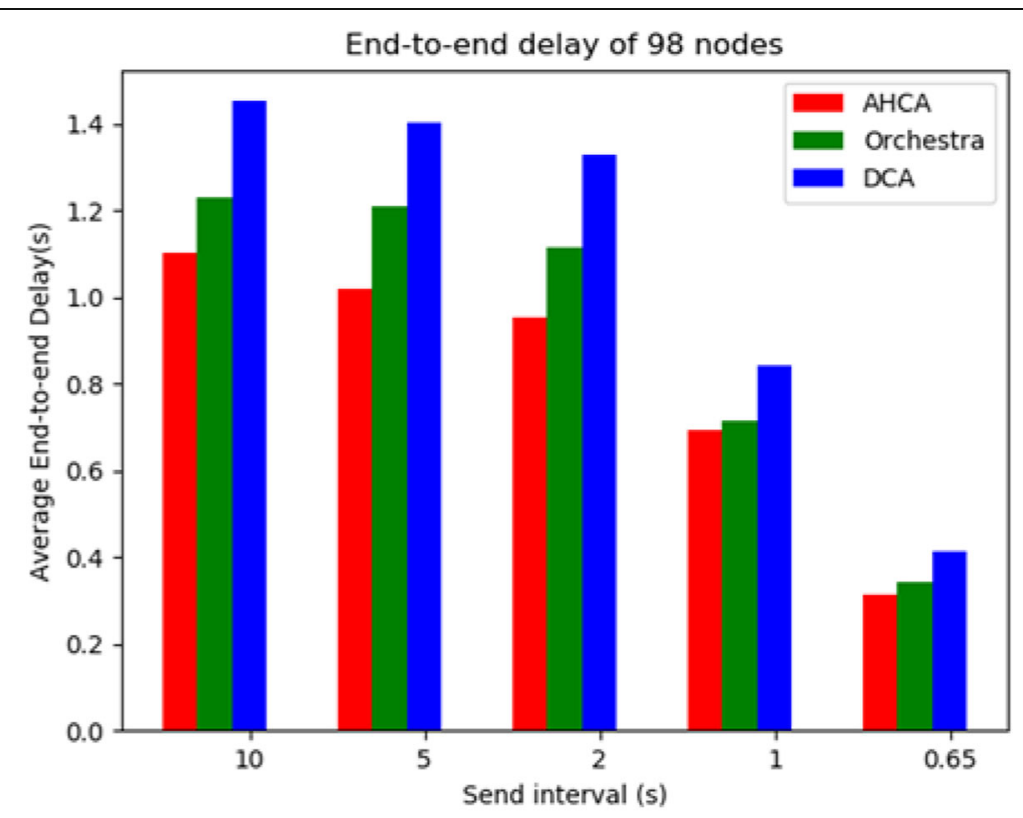

Fig. 7 End-to-end delay of 98 nodes

relation of $Y$ between channel configuration ratio, different channel configuration ratio, volume change, plug rate formula (10), and corresponding enumerated channel configuration ratio static channel, and obtained the following simulation results as shown in Fig. 9 and 10.

According to Figs. 9 and 10, it can be seen that

(1) In the case of less node information transfer, AHCA congestion rate is generally lower;

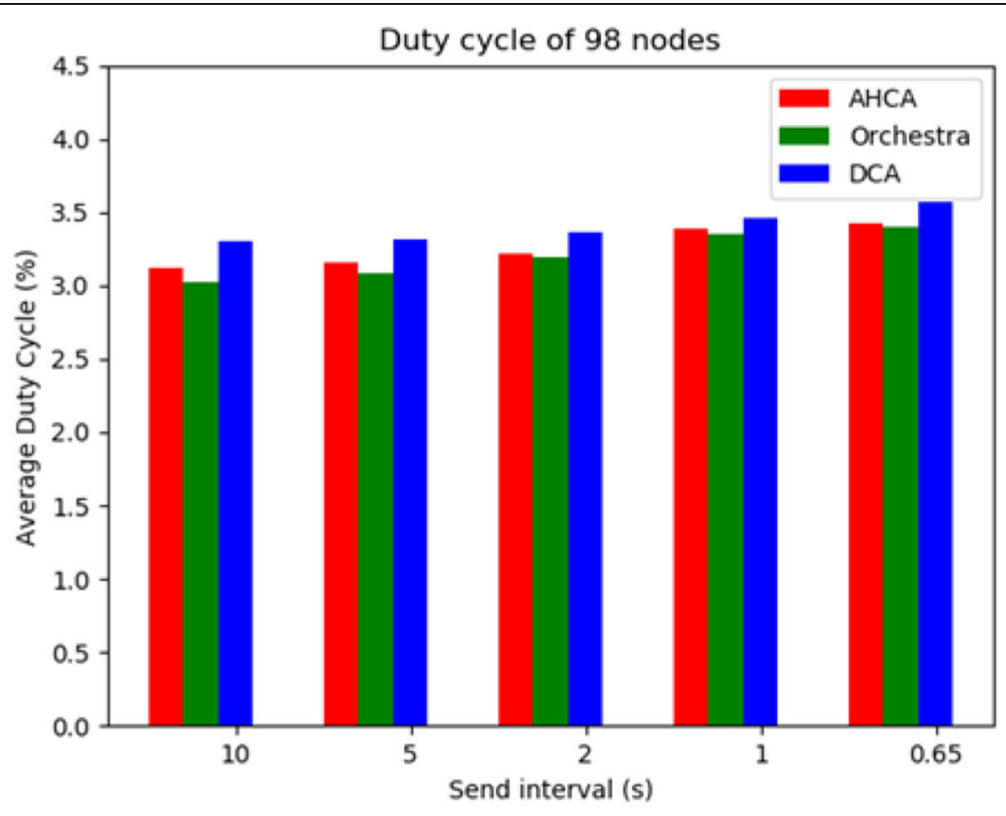

Fig. 8 Duty cycle of 98 nodes 


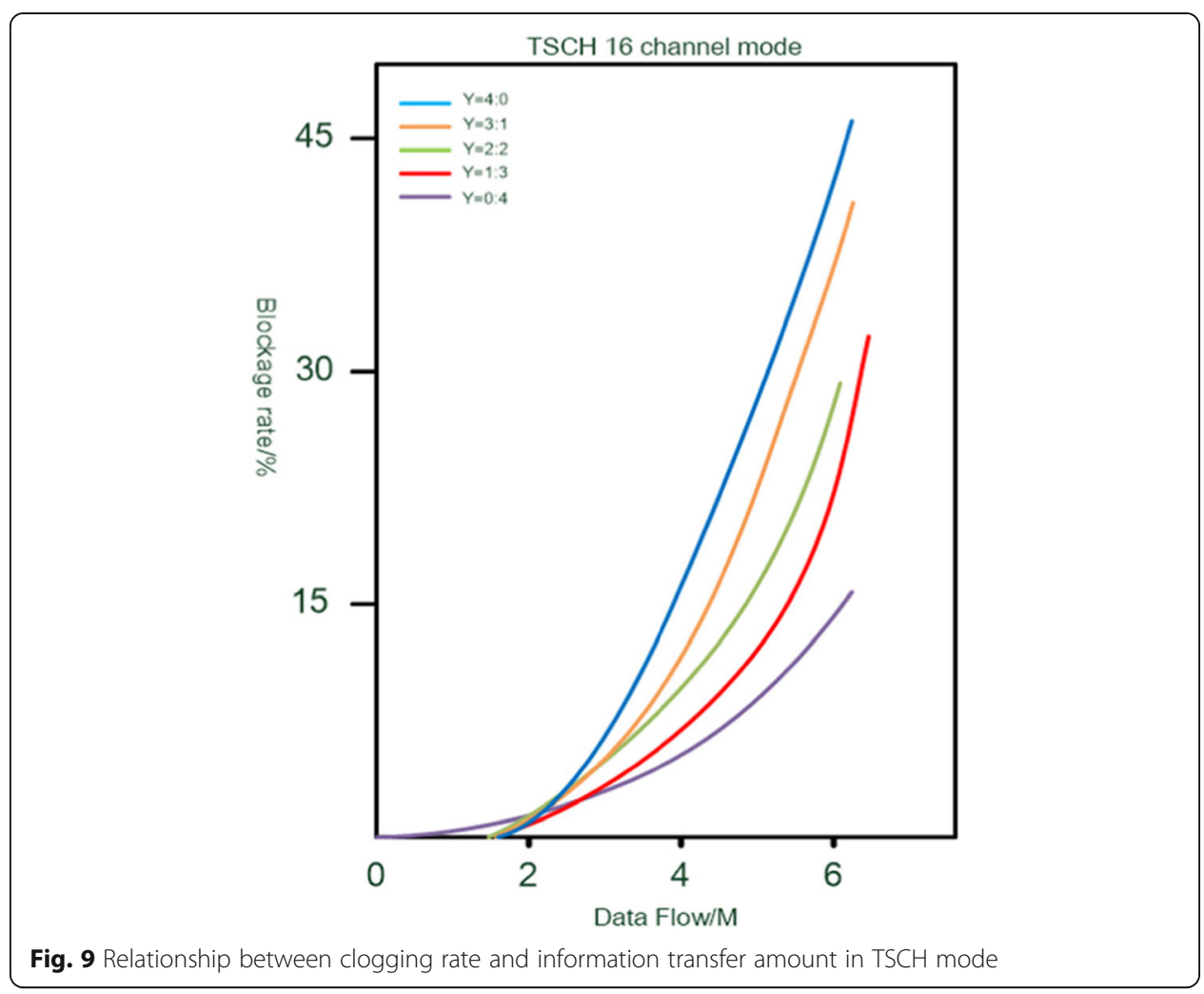

Fig. 9 Relationship between clogging rate and information transfer amount in TSCH mode

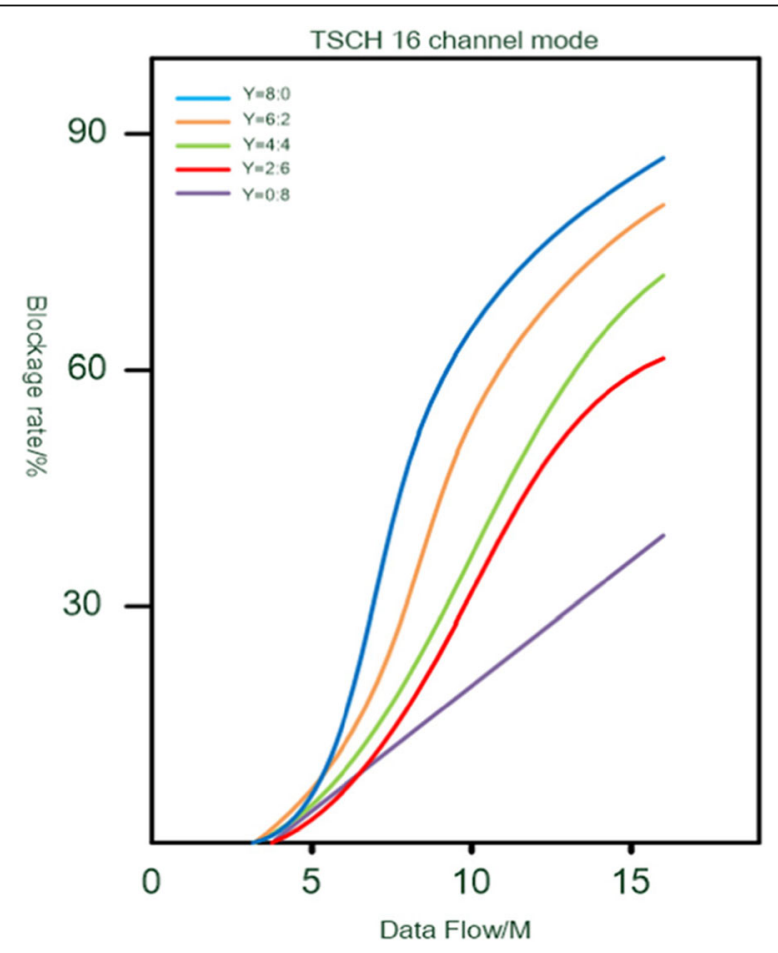

Fig. 10 Relationship between clogging rate and information transfer amount in TSCH mode 
(2) In the case of a large amount of node information transmission, the congestion rate of HCA is generally high. When the data amount is about $5 \mathrm{M}$, when $Y=4: 0$, it reaches its peak at about $46 \%$, while when $Y=0: 4$, the congestion rate is only $18 \%$.

(3) With the increase of information transmission, the AHCA scheme with large $Y$ has better performance.

(4) The total number of channels is constant, and at this time, there is a uniquely determined $Y$ under different information amounts, which minimizes the network congestion rate.

In view of the above conclusions, as long as the amount of information transmitted in the network is counted at any time, the channel allocation can be adjusted to the optimal value according to the proportion to follow the change of the amount of information transmitted.

It can be seen from Figs. 3, 4, and 5 that the adaptive hybrid channel allocation strategy has made good progress in reducing the information transmission time, and from Figs. 6, 7, and 8 that it has significant advantages over the traditional single TSCH frequency-hopping channel dynamic programming and Orchestra algorithm scheduling in reducing end-to-end delay, improving network reliability, and reducing radio load ratio. It can be seen from Figs. 9 and 10 that AHCA schemes with different proportions of public and private channels exhibit different performance characteristics under different information transmission volumes, so it is sometimes impossible to completely guarantee their good performance under different loads.

It is worth noting that our statistical period should be determined according to the variation of the amount of data transmitted in the channel. When the data transmitted in the channel changes dramatically, we need to set a shorter statistical period.

\section{Conclusions and future work}

In this paper, we put forward the channel quality of hybrid channel allocation scheme to fast, quality with channel estimation technology, designed to prolong the life cycle of wireless sensor network (WSN), at the same time reduces the cast time of pseudo random channel frequency hopping, experiments show that compared with the results of other strategies, this paper puts forward the AHCA strategy can increase the reliability of the network at the same time, save the network energy consumption and to reduce the network information transmission time also has a good effect.

Next, we have two works to do. First, we will consider the security into the AHCVTSCH network to improve the reliability of information transmission in the network while reducing the network congestion rate and increasing the network lifetime; secondly, the blacklist mechanism is considered to enter the AHCV-TSCH network, which further reduces the energy consumption required for information transmission between nodes and prolongs the overall life of the network. 
Authors' contributions

All authors discussed the experiment. JXG conducted experiments and wrote papers. Finally, FM, YX, and YJZ published some useful comments in this article. All authors have read and approved the final manuscript.

Funding

This work was partially funded by the Natural Science Foundation of Anhui Province. The Natural Science Foundation of Anhui Province Number: 1908085MF214.

\section{Availability of data and materials}

Not application.

\section{Competing interests}

The authors declare that they have no competing interests.

Received: 27 November 2019 Accepted: 14 August 2020

Published online: 04 September 2020

\section{References}

1. D.D. Guglielmo, A. Seghetti, G. Anastasi, et al., A performance analysis of the network formation process in IEEE 802.15.4e TSCH wireless sensor/actuator networks (2014), pp. 1-6

2. Elsts, Atis, et al. Scheduling high-rate unpredictable traffic in IEEE 802.15.4 TSCH networks. International Conference on Distributed Computing in Sensor Systems 2018.

3. Ben Yaala, Sahar F. Theoleyre, and R. Bouallegue. [IEEE 2018 IEEE 32nd International Conference on Advanced Information Networking and Applications (AINA) - Krakow, Poland (2018.5.16-2018.5.18)] 2018 IEEE 32nd International Conference on Advanced Information Networking and Applications (AINA) - Experimental Analysis of the Efficiencyof Shared Access in IEEE802.15.4-TSCH Networks with Sporadic Traffic. " 2018:9-16.

4. Elsts, Atis, et al. Adaptive channel selection in IEEE 802.15.4 TSCH networks. Global Internet of Things Summit IEEE, 2017.

5. Ojo, Mike, et al. An energy efficient centralized scheduling scheme in TSCH networks. 2017 IEEE International Conference on Communications Workshops (ICC Workshops) IEEE, 2017.

6. R.D. Gomes et al., in Personal, Indoor, and Mobile Radio Communication (PIMRC), 2014 IEEE 25th Annual International Symposium on IEEE. Distributed approach for channel quality estimation using dedicated nodes in industrial WSN (2014)

7. K.T. Kim et al., in 2017 19th International Conference on Advanced Communication Technology (ICACT). An industrial IoT MAC protocol based on IEEE 802.15.4e TSCH for a large-scale network (2017)

8. A. Aijaz, U. Raza, DeAMON: a decentralized adaptive multi-hop scheduling protocol for $6 \mathrm{TiSCH}$ wireless networks. IEEE Sensors J. 99, 1-1 (2017)

9. P.H. Gomes, T. Watteyne, B. Krishnamachari, MABO-TSCH: Multihop and blacklist-based optimized time synchronized channel hopping. Transactions on Emerging Telecommunications Technologies 1 (2017), p. e3223

10. T. Hamza, G. Kaddoum, Enhanced minimal scheduling function for IEEE 802.15.4e TSCH networks. IEEE Wireless Communications and Networking Conference ( IEEE WCNC) IEEE (2019)

11. Y. Sun, L. Zhang, G. Feng, B. Yang, B. Cao, M.A. Imran, Blockchain-enabled Wireless Internet of Things: performance analysis and optimal communication node deployment. IEEE Intern. Things J. 6(3), 5791-5802 (2019)

12. X. Vilajosana, K. Pister, Minimal 6TSCH Configuration (2013)

13. M. Benaddy, B.E. Habil, O.E. Meslouhi, et al.,A mutlipath routing algorithm for wireless sensor networks under distance and energy consumptionconstraints for reliable data transmission. International Conference on Engineering \& Mis IEEE, 2018

14. W. Yang et al., Industrial loT secure time synchronization strategy based on IEEE802.15.4e standard. Comput. Res. Dev. 9,720-724 (2017)

15. A. Shafieinejad, F. Hendessi, F. Fekri, Star-structure network coding for multiple unicast sessions in wireless mesh networks. Wireless Personal Communications 72(4), 2185-2214 (2013)

16. D. Xingjian, S. Guodong, G. Yang, et al., Link Investigation of IEEE 802.15.4 Wireless Sensor Networks in Forests. Sensors 16(7), 987-994 (2016)

17. B. Pavkovic, A. Duda, W.-J. Hwang, et al., Efficient topology construction for RPL over IEEE 802.15 .4 in wireless sensor networks. Ad. Hoc. Netw. 15(apr.), 25-38 (2014)

\section{Publisher's Note}

Springer Nature remains neutral with regard to jurisdictional claims in published maps and institutional affiliations. 